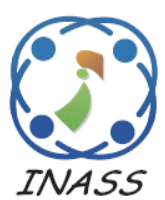

\title{
Dispatching Model in Online Taxi System by Using Stable Marriage and First Price Sealed Bid Auction Models
}

\author{
Purba Daru Kusuma ${ }^{1 *} \quad$ Meta Kallista $^{1}$ \\ ${ }^{1}$ Computer Engineering, Telkom University, Indonesia \\ * Corresponding author's Email: purbodaru @telkomuniversity.ac.id
}

\begin{abstract}
Dispatching system plays a crucial role in today smartphone based online taxi system. There are three common models that are used in online taxi dispatch system: (1) first come first served, (2) stable marriage, and (3) agent-based models. Unfortunately, these existing models accommodate passenger's waiting time and/or driver's pickup cost only. Meanwhile, there are several other important aspects: price, driver's service level, and passenger's behaviour. Based on this problem, this work aims to develop a dispatching model for online taxi system that accommodates these aspects too rather than waiting time and pickup cost only. This model is developed by combining stable marriage and first price sealed bid auction. The stable marriage is used to satisfy both passenger and driver. The first price sealed bid auction is used to offer a competitive price for the passenger that is still accepted by the driver. Based on the simulation result, overall, the proposed model performs the best among the previous models, except in the average pickup distance. In the extreme undersupply condition, the proposed model creates 20 percent higher in the average customer's satisfaction score and equal in the average net distance. In the extreme oversupply condition, the proposed model creates 28 percent higher in the vehicle's satisfaction score. The proposed model creates up to 21 percent lower travel cost. Although the proposed model creates the worst in the average pickup distance, the average pickup time is 3.9 minutes with the assumption that the vehicle's speed is $10 \mathrm{~km}$ per hour.
\end{abstract}

Keywords: Dispatching model, Online taxi, Stable marriage, First price sealed bid auction.

\section{Introduction}

Nowadays, online taxi system becomes very popular. This condition is catalysed by the smartphone technology which the global positioning system (GPS) receiver is embedded [1]. By using GPS equipped smartphone, destination, and vehicle current locations are easily and accurately provided. This system is more efficient due to reducing drivers' time and energy and passengers' expense [2].

One important system in the online taxi system is the dispatching system. The role of the dispatching system is allocating passenger booking request to the preferred vehicle [3]. There are many studies conducted on the dispatching system in the online taxi system. They can be grouped into three approaches. The first approach is the combination between first come first served (FCFS) and the nearest driver models [4]. The second one is the stable marriage model [5]. The first model has been proven in achieving local optimal [6] while the second one has been proven in achieving global optimal and fairness [7]. The third one is the agentbased model [8]. This third model is the most widely used model in the latest taxi dispatching works.

Unfortunately, many of these studies in the dispatching system used only distance or time between the pickup and the current vehicle locations in matching the request and the vehicle. It occurs in studies that adopted either FCFS [4], stable marriage [5], or agent-based model [9]. The reason is the only aspect that is concerned by the passenger is waiting time and the only aspect that is concerned by the driver is the pickup cost [9].

Although these two aspects are important, there are several other aspects that become concerned in both passenger and driver. Passenger also concerns about driver's attitude and vehicle condition [10]. 
Drivers' driving attitude may affect the travel safety [11]. Meanwhile, the driver also concerns about revenue [12] and passenger's attitude. In the current online taxi systems, such as Go-Jek, Grab, or Uber, both drivers and passengers can assess their counterpart by giving a score based on their satisfaction [13]. Moreover, the company is aware of this assessment to maintain both passengers' and drivers' satisfaction so that they will keep engaged in the system [2].

Nowadays, online taxi price is dynamic based on the supply-demand balance [13]. In the overdemand situation, the price rises and on the other side, in the oversupply situation, the price goes down. For example, Uber charges premium price during peak hours and give discount during off-peak [14]. UberX also applies different base fare index based on the city in the Unites States of America [15]. This dynamic occurs in all vehicles. Moreover, in the same supplydemand balance, all vehicles get the same price. In the collaborative online taxi system where the drivers are not the employee of the company, there are various vehicle types with various car price range.

Due to this uniform price treatment among drivers, there is no incentive for drivers to improve their service level. The driver tends to choose cheaper vehicle rather than the more expensive and sophisticated vehicle. There is also no price incentive for drivers to improve their driving behaviour. In general, drivers, behave based on incentive [13], especially price incentive. Unfortunately, this individual price incentive has not been accommodated in the either existing online taxi dispatch system or in the online taxi dispatch studies.

Based on this problem, in this work, we propose a novel online taxi dispatching model that accommodates not just waiting time or pickup cost but also individual price incentive. This model is developed based on stable marriage model. This model is combined with the first price sealed bid (first price) auction in the simultaneous bidding process. The first price auction is very popular in electronic auction [16] Auction mechanism is adopted to give price incentive for passenger and better winning opportunity for the driver who submits lower bidding price although it is not the only accommodated variable. As a negotiation mechanism, the bidding price ranges between driver's individual target point and reservation price.

Our contributions on this paper are as follows.

(1) We accommodate passenger satisfaction, driver satisfaction, and financial incentive into the online taxi dispatching system rather than waiting time and pickup cost only as in the existing dispatching model.
(2) We introduce an individual price mechanism rather than an existing uniform price mechanism as in the existing online taxi system and it is accommodated in the proposed dispatching system.

The remainder of this paper is structured as follows. In the second section, we explain the related work to strengthen the necessity of this work and the reasoning of the chosen methods. The topics in the second section include: online taxi dispatch system, stable marriage model, and first price sealed bid auction. In the third section, we explain the models that include the mathematical model and the simulation model. In the fourth section, we show the simulation result and discuss the findings. In the fifth section, we conclude the work by connecting the research purpose, model, and result findings.

\section{Related work}

\subsection{Online taxi dispatch system}

Nowadays, due to the massive penetration of smartphone technology, the taxi system also evolves. Uber, DiDi Chuxing, and Lyft have transformed the way people travel [17]. In the current online taxi system, passengers and drivers are connected to the system by using their smartphone. New online taxi companies, such as Grab, Go-Jek, and Uber disrupt the traditional taxi industry [14]. In China, service order of the conventional cruising taxi fell at least 35 percent [18]. This new system improves the service quality because information about passengers and vehicles' locations are well provided. In this online taxi system, a dispatching system to match between passengers and drivers becomes crucial.

There are several popular methods in the online taxi dispatching system. The first method is the combination between FCFS and the nearest driver. In this model, every time a new order request arrives, the system will process this request immediately [19]. The system will find the available vehicles and or will soon available vehicles near the pickup location. Then, this request will be allocated to the nearest vehicles to achieve the lowest passenger's waiting time [19]. Kusuma proposed the combination between the FCFS, the nearest driver, and first-infirst-out model [4]. In this work, the driver's idle time is also concerned so that drivers with the longer idle time are prioritized. This work has been improved in the later work by adding the first price auction mechanism where drivers in the passenger's observation range can bid price to win the order [20]. The goal of this work is to introduce the price competition so that it can reduce the passenger's travel cost [20]. This work showed that by 


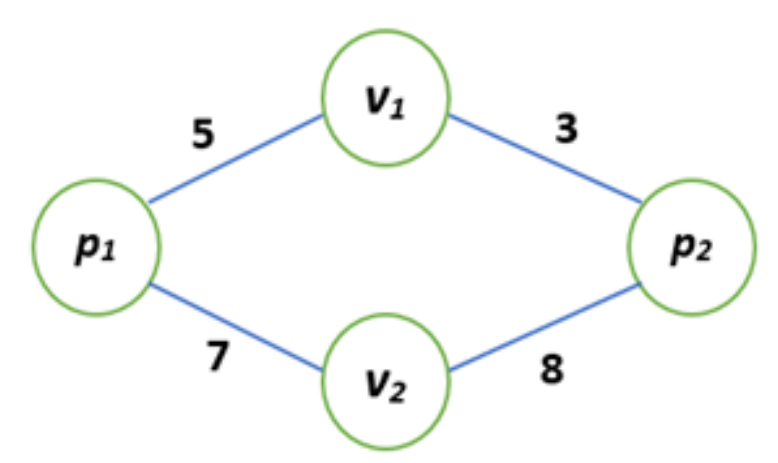

Figure. 1 Matching illustration

implementing price competition, the total travel cost for 100 orders is only 13.8 percent rather than without price competition.

The second model is the stable marriage model. In this model, there is a dispatching time interval [5]. In every interval or time window, incoming booking requests are pooled first rather than are immediately dispatched so that there are more requests and available taxis within the time window [5].

By using stable marriage, passengers' preference will be matched with the drivers' preference. The illustration of this problem is shown in Fig. 1.

In Fig. 1, there are two passengers, $p_{1}$ and $p_{2} . p_{1}$ arrives earlier than $p_{2}$. Meanwhile, in the system, there are two available vehicles, $v_{1}$ and $v_{2}$. If the system implements FCFS, then $p_{1}$ will be served by $V_{1}$ because $p_{1}$ is closer to $V_{1}$ rather than $V_{2}$. Because $V_{2}$ is the only available vehicle then $p_{2}$ will be served by $V_{2}$. Based on this model, the total cost is 13 . If the system implements stable marriage, there are two scenarios: vehicle submission and passenger submission. In the vehicle submission, vehicles submit proposal and passengers evaluate proposal. Meanwhile, in the passenger submission, passengers submit passenger and vehicles evaluate proposal. The scenario in the vehicle submission is as follows. First, $V_{1}$ submits proposal to $p_{2}$ and $V_{2}$ submits proposal to $p_{1}$. Because both passengers receive only one proposal each, then both passengers accept their received proposal immediately. So, $p_{1}$ will be served by $V_{2}$ and $p_{2}$ will be served by $V_{1}$. The total cost is 10 . The process in the passenger submission model is as follows. First, both passengers submit to $v_{1}$. Then, $v_{1}$ accepts $p_{2}$ and rejects $p_{1}$. In the second round, $p_{1}$ submits to $V_{2}$ and because $V_{2}$ receives one proposal only, then $V_{2}$ accepts $p_{1}$. The total cost is also 10 . Based on this simple simulation, it shows that the total cost in stable marriage system is lower than in FCFS system.

The third model is the agent-based model. This model is widely used in the several latest online taxi dispatch studies. These several studies will be discussed below.

The first agent-based dispatch model can be called as limited-movement-grid (LMG) model [9]. In it, the map is divided into square-shaped grids with specified size. The drivers become the agents. The shortest distance is adopted in this model. The agent prioritizes passengers in the same grid first. If in the single grid, the number of drivers is equal to or less than the number of the passengers then every driver will get the nearest passenger inside the grid. Otherwise, the drivers that fail to get a passenger in their own grid can move to the left, right, up, or down for one grid. In this work, dispatching parameters are passenger waiting time and vehicle pickup distance. This dispatching model is then combined with the pre-dispatching model to improve the supply-demand balance.

The second agent-based dispatch model can be called as a bipartite matching model [8]. In it, both passengers and drivers become autonomous agents that can interact with each other. In this model, the Hungarian method as a bipartite matching model is implemented to achieve global optimization. The concerned parameter is the minimum cost of the passenger's perspective (pickup distance or waiting time). The Euclidean distance is used in measuring the distance between passengers and drivers.

The third agent-based dispatch model can be called as free-movement-grid (FMG) model [21]. In it, the grids represent the agents. So, the main goal is maximizing the revenue of the grid. In the first step, orders in every grid are sorted based on the revenue. In this work, the revenue consists of two revenues: current revenue and potential revenue. The current revenue is revenue which is grabbed from the current order. The potential revenue is the revenue that may be grabbed in the area surrounding the current order destination. Orders with higher revenue will be prioritized. In every order matching, the order will be matched to the nearest available driver in the same grid. In the second step, the unexecuted orders will be sorted again based on the revenue without concerning the grid. Once again, higher revenue orders will be prioritized. Every order will be allocated to the nearest available driver.

The fourth agent-based dispatch model can be called as circle-shaped-maximum-revenue (CSMR) model [17]. In it, agents are represented by the drivers. Rather than using square-shaped grid, circle-shaped agent's observation area with a specified radius is used. This dispatch model also focuses on improving revenue, which is the trade-off between short-term revenue (revenue per order) and long-term revenue (daily revenue). The driver will bid order with the 
highest revenue or travel distance. In the case that there are some drivers who bid the same passenger, the order will be matched to the nearest driver.

The fifth agent-based dispatch model can be called as circle-shaped-nearest (CSN) model [22]. In it, the drivers represent the agents too. The circleshaped observation range is also used. In this model, every vehicle prioritizes the nearest distance to minimize the empty driving distance. In case there are some drivers bid the same passenger, vehicle with the shortest distance is prioritized.

\subsection{Stable marriage}

Stable marriage algorithm is a stable matching algorithm or model which was first introduced by Gale and Shapley in 1962 which was implemented in the college admission system [23]. This algorithm was developed based on submission and acceptance. The system consists of $n$ men and $n$ women where men submit proposal and women accept or reject proposal. The role can be interchanged. It was proven that there is at least one stable solution [23].

The process of this basic stable marriage algorithm is as follows [23]. This algorithm consists of iterations or rounds which stop after stable pairs situation is achieved. Each round consists of a submission and acceptance session. In the submission session, all unengaged men submit proposal to their most preferred woman with the condition that every man never submits to the woman whom he ever submits. In the acceptance session, all women evaluate the received proposals, accept the most preferred one, and reject the others. The men whose proposal is accepted then become engaged while the women who receive proposal become engaged too. In the next round, the engaged men do not submit proposal. Meanwhile, engaged women still can receive proposal. If the women receive a better proposal, then they accept the newer proposal and engage with the new men. The previous men whose proposal is declined become unengaged and can submit a new proposal in the next round. In the condition that the number of men is equal to the number of women, iteration stops when the last woman receives and accepts proposal. It means that there no change in pairs anymore. This algorithm is also easily implemented in the scenario where the number of men is not equal to the number of women.

\subsection{First price sealed bid auction}

Auction is one effective method in allocating or distributing limited resources to the parties [24]. Auction is also a common mechanism to sell products that are not easy to value [16]. This method is popular from the Sotheby art stuff, Dutch flower market, real estate, government procurement, to the e-commerce era. This is popular due to its method of finding party that can offer the best yield.

Auctions can be grouped into two types: sealedprice auction and bilateral auction price (open-price auction) [25]. The sealed-price auction includes the first price sealed-bid auction and the second price sealed-bid auction. Meanwhile, the open-price auction includes the English auction and the Dutch auction. In the sealed-bid auction, the bid price remains secret. It only reveals the winning price. The sealed-price auction is better in maintaining bidders' privacy and the system efficiency [16].

As a matching method, first price auction can also be implemented in online taxi system where every driver has his price range. The goal is offering the passenger with a competitive service price that is still accepted by the winning driver.

\section{Research method}

\subsection{Model}

This dispatching model is basically developed based on the stable marriage model. As a stable marriage, there two consecutive processes: proposal submission and proposal acceptance. In this work, proposal is submitted by the driver. On the other side, proposal acceptance is conducted by the passenger or customer. Inside the proposal, every driver also bids the service price. Due to the adoption of first price sealed bid auction model, a driver cannot observe their opponents' bidding price. In this model, the passenger term has the same meaning as the customer term.

In this model, several notations are used. These notations are as follows.

$n_{V} \quad$ number of vehicles

$n_{c} \quad$ number of customers

$n_{c s} \quad$ number of closed customers

$i \quad$ vehicle index

$j \quad$ customer index

$k \quad$ price index

$I_{p, j} \quad$ customer's pickup location

$I_{d, j} \quad$ customer's destination location

$I_{i} \quad$ vehicle's current location

$d_{p, i, j}$ pickup distance between vehicle and customer

$d_{d, i j} \quad$ vehicle driving distance in serving customer

$d_{t, j} \quad$ customer's travel distance

$d_{n, i, j} \quad$ vehicle's net distance

$d_{a v p}$ average pickup distance

$d_{a v n}$ average net distance

$d_{n i, i} \quad$ vehicle's minimum net distance

$d_{n a, i}$ vehicle's maximum net distance 
$d_{p i j} \quad$ customer's minimum pickup distance

$d_{p a j}$ customer's maximum pickup distance

$m_{i, j} \quad$ vehicle-customer link value

$p_{t, i} \quad$ vehicle's target point

$p_{r, i} \quad$ vehicle's reservation price

$p_{r i} \quad$ minimum reservation price

$p_{\text {mid }}$ medium price

$p_{t a} \quad$ maximum target point

$p_{b, i} \quad$ vehicle's bidding price

$p_{\text {step }}$ step price

$p_{\text {atc }}$ average travel cost

$p_{c, i j} \quad$ customer's cost by using vehicle

$p_{c, j} \quad$ customer's closed price

$p_{s, j, k} \quad$ submitted price to customer

$P_{s, j} \quad$ Set of distinct prices that are submitted to customer and they are sorted ascendingly

$W_{n} \quad$ net distance weight

$W_{p}$ pickup distance weight

$W_{b}$ bidding price weight

$W_{V} \quad$ vehicle satisfaction weight

$W_{c} \quad$ customer satisfaction weight

$s_{V, i j}$ vehicle's total score from customer's perspective

$s_{p, i j}$ vehicle's pickup distance score from customer's perspective

$s_{b, i j} \quad$ vehicle's bidding price score from customer's perspective

$S_{v i, j}$ vehicle's satisfaction score from customer's perspective

$S_{a v} \quad$ average vehicle satisfaction score

$S_{w p, i j}$ vehicle's weighted pickup distance score from customer's perspective

$S_{w b, i, j}$ vehicle's weighted bidding price score from customer's perspective

$S_{W v i, j}$ vehicle's weighted satisfaction score from customer's perspective

$S_{c, i j}$ customer's total score from vehicle's perspective

$S_{n, i j} \quad$ customer's net distance score from vehicle's perspective

$S_{c, i j}$ customer's satisfaction score from vehicle's perspective

$S_{a c} \quad$ average customer satisfaction score

$S_{w n, i j}$ customer's weighted net distance score from vehicle's perspective

$S_{w c, i j}$ customer's weighted satisfaction score from vehicle's perspective

$S_{c i, i}$ customer minimum satisfaction score from vehicle's perspective

$s_{c a, i}$ customer maximum satisfaction score from vehicle's perspective

$S_{V i j}$ vehicle minimum satisfaction score from customer's perspective
$S_{v a, j}$ vehicle maximum satisfaction score from customer's perspective

$S_{b i j} \quad$ minimum bidding price received by customer

$S_{b a, j}$ maximum bidding price received by customer

$S_{p r, j}$ set of vehicles that send proposal to customer

$S_{c u, i}$ set of customers that have been proposed by vehicle

$S_{l, i, j} \quad$ set of links between selected vehicle and selected customer

$c_{p r o, j}$ customer that is proposed by vehicle

$V_{s, j} \quad$ vehicle that is selected by customer

$S_{t v, i} \quad$ vehicle's status

$s_{t c, j} \quad$ customer's status

$s t_{m} \quad$ matching process status

$s t_{s} \quad$ stable status

$s t_{m y}$ vehicle matching status

$s t_{m c} \quad$ customer matching status

$t_{b, i} \quad$ vehicle's bidding time

$t_{s, j} \quad$ customer's selection time

$r_{s u} \quad$ success ratio

$U()$ uniform distribution random function

\section{Algorithm 1: Matching process}

$s t_{m}=0$

while $s t_{m}=0$ do

begin

proposal_submission()

proposal_selection()

stable_checking()

end

There are three types of time that are used in this model: iteration time, vehicle's bidding time, and passenger's selection time. These times are discrete. The iteration time increments when the three processes in single iteration are complete. The vehicle's bidding time increments every time a vehicle submits a proposal. The vehicle's bidding time may be different among vehicles because in single iteration time, some vehicles may submit a proposal while the others may not. The passenger's selection time increments every time a passenger evaluates proposal or proposals. The passenger's selection time may be different among passengers because in single iteration time, some passengers may evaluate proposals while the others may not because they do not receive proposal.

The first process is the proposal submission process. In this process, all unengaged vehicles send proposal to their most prioritized customer. There are two parameters that are concerned by the vehicle in determining his most prioritized customer: net distance and customer satisfaction. This customer selection is formalized by using Eqs. (1) to (14). 


$$
\begin{gathered}
d_{p, i, j}=\left\|l_{p, j}-l_{i}\right\| \\
d_{t, j}=\left\|l_{d, j}-l_{p, j}\right\| \\
d_{d, i, j}=d_{p, i, j}+d_{t, j} \\
d_{n, i, j}=d_{t, j}-d_{p, i, j} \\
d_{n i, i}\left(t_{b, i}\right)=\min \left(d_{n, i, j}\right) \wedge j \notin S_{c u, i}\left(t_{b, i}\right) \\
d_{n a, i}\left(t_{b, i}\right)=\max \left(d_{n, i, j}\right) \wedge j \notin S_{c u, i}\left(t_{b, i}\right) \\
s_{n, i, j}\left(t_{b, i}\right)=\left\{\begin{array}{l}
1, d_{n i, i}\left(t_{b, i}\right)=d_{n a, i}\left(t_{b, i}\right) \\
\frac{d_{n, i, j}-d_{n i, i}\left(t_{b, i}\right)}{d_{n a, i}\left(t_{b, i}\right)-d_{n i, i}\left(t_{b, i}\right)}, \text { else }
\end{array}\right.
\end{gathered}
$$

The explanation of the Eqs. (1) to (7) is as follows. Euclidean distance is used in determining the pickup distance as in Eq. (1) and the travel distance as in Eq. (2). In Eq. (3), the driving distance is the summation of the pickup distance and the travel distance. In Eq. (4), the net distance is the difference between the travel distance and the pickup distance. In Eqs. (5) and (6), the minimum and maximum net distance are calculated among customers whom they have not been submitted to. These minimum and maximum net distance scores are used to normalize the net distance. Eq. (7) shows that the net distance is proportional to the net distance score.

$$
\begin{gathered}
s_{c i, i}\left(t_{b, i}\right)=\min \left(s_{c, j}\right) \wedge j \notin S_{c u, i}\left(t_{b, i}\right) \\
s_{c a, i}\left(t_{b, i}\right)=\max \left(s_{c, j}\right) \wedge j \notin S_{c u, i}\left(t_{b, i}\right) \\
s_{c, i, j}\left(t_{b, i}\right)=\left\{\begin{array}{l}
1, c_{c i, i}\left(t_{b, i}\right)=c_{c a, i}\left(t_{b, i}\right) \\
\frac{s_{c, j}-s_{c i, i}\left(t_{b, i}\right)}{s_{c a, i}\left(t_{b, i}\right)-s_{c i, i}\left(t_{b, i}\right)}, \text { else }
\end{array}\right.
\end{gathered}
$$

The explanation of Eqs. (8) to (10) is as follows. The minimum customer's satisfaction score is formalized by using Eq. (8), while the maximum customer's satisfaction score is formalized by using Eq. (9). These minimum and maximum scores are used to normalize the customer's satisfaction score. The customer's satisfaction score is formalized by using Eq. (10).

$$
\begin{gathered}
s_{w n, i, j}\left(t_{b, i}\right)=w_{n} \cdot s_{n, i, j}\left(t_{b, i}\right) \\
s_{w c, i, j}\left(t_{b, i}\right)=w_{c} \cdot s_{c, i, j}\left(t_{b, i}\right) \\
s_{c, i, j}\left(t_{b, i}\right)=s_{w n, i, j}\left(t_{b, i}\right)+s_{w c, i, j}\left(t_{b, i}\right)
\end{gathered}
$$

$$
c_{p r o, i}\left(t_{b, i}\right)=j, \max \left(s_{c, i, j}\left(t_{b, i}\right)\right)
$$

These scores are weighted by using Eq. (11) for the net distance score and Eq. (12) for the customer's satisfaction weighted score. Then these scores are accumulated by using Eq. (13). Eq. (14) is used to guarantee that the proposal will be submitted to the passenger with the highest accumulated score, and he has not been submitted yet by the vehicle.

Vehicle sends proposal with certain bidding price. This bidding price ranges from his target point and reservation price. In the beginning, the vehicle will bid at its target point. Every time the vehicle sends bidding, his bidding price goes lower until his reservation price. This vehicle's price dynamics is formalized by using Eqs. (15) and (16). Eq. (15) is used when the vehicle bidding time is higher than 0 and Eq. (16) is used to determine initial bidding time.

$$
\begin{aligned}
& p_{b, i}\left(t_{b, i}\right)=\left\{\begin{array}{c}
p_{b, i}\left(t_{b, i}-1\right), p_{b, i}\left(t_{b, i}\right)>p_{r e, i} \\
p_{r, i}, p_{b, i}\left(t_{b, i}\right) \leq p_{r, i}
\end{array}\right. \\
& p_{b, i}(0)=p_{t, i}
\end{aligned}
$$

The second process is the proposal acceptance process. This process is held by the customers. There are three parameters that are concerned by the customer: pickup distance, bidding price, and vehicle satisfaction. This process is formalized by using Eqs. (17) to (31).

$$
\begin{aligned}
& d_{p i, j}\left(t_{s, j}\right)=\min \left(d_{p, i, j}\right) \wedge i \in S_{p r, j}\left(t_{s, j}\right) \\
& d_{p a, j}\left(t_{s, j}\right)=\max \left(d_{p, i, j}\right) \wedge i \in S_{p r, j}\left(t_{s, j}\right) \\
& s_{p, i, j}\left(t_{s, j}\right)=\left\{\begin{array}{c}
1, d_{p i, j}\left(t_{s, j}\right)=d_{p a, j}\left(t_{s, j}\right) \\
1-\frac{d_{p, i, j}-d_{p i, j}\left(t_{s, j}\right)}{d_{p a, j}\left(t_{s, j}\right)-d_{p i, j}\left(t_{s, j}\right)}, \text { else }
\end{array}\right.
\end{aligned}
$$

From the customer's perspective, pickup distance aspect is formalized by using Eqs. (17) to (19). Among the received proposals, Eq. (17) is used to calculate the minimum pickup distance, and Eq. (18) is used to calculate the maximum pickup distance. These minimum and maximum scores are used to normalize the pickup distance. Then, Eq. (19) is used to determine the pickup distance score of the received proposal. Eq. (19) shows that the pickup distance is inversely proportional to the pickup distance score.

$$
\begin{gathered}
s_{v i, j}\left(t_{s, j}\right)=\min \left(s_{v, i}\right) \wedge i \in S_{p r, j}\left(t_{s, j}\right) \\
s_{v a, j}\left(t_{s, j}\right)=\max \left(s_{v, i}\right) \wedge i \in S_{p r, j}\left(t_{s, j}\right)
\end{gathered}
$$




$$
s_{v, i, j}\left(t_{s, j}\right)=\left\{\begin{array}{l}
1, s_{v i, j}\left(t_{s, j}\right)=s_{v a, j}\left(t_{s, j}\right) \\
\frac{s_{v, i}-s_{v i, j}\left(t_{s, j}\right)}{s_{v a, j}\left(t_{s, j}\right)-s_{v i, j}\left(t_{s, j}\right)}, \text { else }
\end{array}\right.
$$

From the customer's perspective, vehicle satisfaction aspect is formalized by using Eq. (20) to Eq. (22). Among the received proposals, Eq. (20) is used to calculate the minimum vehicle satisfaction score, and Eq. (21) is used to calculate the maximum vehicle satisfaction score. These minimum and maximum scores are used to normalize the vehicle satisfaction score. Then, Eq. (22) is used to determine the vehicle satisfaction score of the received proposal.

$$
\begin{aligned}
& s_{b i, j}\left(t_{s, j}\right)=\min \left(p_{b, i}\left(t_{s, j}\right)\right) \wedge i \in S_{p r, j}\left(t_{s, j}\right) \\
& s_{b a, j}\left(t_{s, j}\right)=\max \left(p_{b, i}\left(t_{s, j}\right)\right) \wedge i \in S_{p r, j}\left(t_{s, j}\right) \\
& s_{b, i, j}\left(t_{s, j}\right)=\left\{\begin{array}{c}
1, s_{b i, j}\left(t_{s, j}\right)=s_{b a, j}\left(t_{s, j}\right) \\
1-\frac{p_{b, i}\left(t_{s, j}\right)-s_{b i, j}\left(t_{s, j}\right)}{s_{b a, j}\left(t_{s, j}\right)-s_{b i, j}\left(t_{s, j}\right)}, \text { else }
\end{array}\right.
\end{aligned}
$$

From the customer's perspective, the bidding price aspect is formalized by using Eqs. (23) to (25). Among the received proposals, Eq. (23) is used to calculate the minimum bidding price score, and Eq. (24) is used to calculate the maximum bidding price score. These minimum and maximum price scores are used to normalize the bidding price. Then, Eq. (25) is used to determine the vehicle bidding price score of the received proposal. Eq. (25) shows that the bidding price is inversely proportional compared to the bidding price score.

$$
\begin{gathered}
s_{w p, i, j}\left(t_{s, j}\right)=w_{p} \cdot s_{p, i, j}\left(t_{s, j}\right) \\
s_{w v, i, j}\left(t_{s, j}\right)=w_{v} \cdot s_{v, i, j}\left(t_{s, j}\right) \\
s_{w b, i, j}\left(t_{s, j}\right)=w_{b} \cdot s_{b, i, j}\left(t_{s, j}\right) \\
s_{v, i, j}\left(t_{s, j}\right)=s_{w p, i, j}\left(t_{s, j}\right)+s_{w v, i, j}\left(t_{s, j}\right)+ \\
s_{w b, i, j}\left(t_{s, j}\right) \\
v_{s e l, j}\left(t_{s, j}\right)=i, \max \left(s_{v, i, j}\left(t_{s, j}\right)\right)
\end{gathered}
$$

$p_{c, j}\left(t_{s, j}\right)=$

$\left\{\begin{array}{c}p_{s, j, 1}\left(t_{s, j}\right), p_{s, j, 1}\left(t_{s, j}\right) \geq p_{r, i} \wedge i=v_{s, j}\left(t_{s, j}\right) \\ p_{r, i}, p_{s, j, 1}\left(t_{s, j}\right)<p_{r, i} \wedge i=v_{s, j}\left(t_{s, j}\right)\end{array}\right.$

These three scores then are weighted based on the prioritization. The weighing process is formalized by using Eqs. (26) to (28) for the pickup distance score, vehicle satisfaction score, and bidding price score consecutively. Then, these weighted scores are accumulated by using Eq. (29). Eq. (30) shows that the winning vehicle is vehicle with the highest cumulative score. Eq. (31) shows that the closed price cannot be lower than the selected driver's reservation price.

This matching process is formalized by the link between vehicle $i$ and customer $j$. If the link value is 1 then vehicle $i$ is matched with customer $j$. Otherwise, the link value is 0 . This process is formalized by using Eq. (32). The link value then is used to determine the status of vehicle and customer as shown in Eqs. (33) and (34).

$$
\begin{gathered}
m_{i, j}\left(t_{i}\right)=\left\{\begin{array}{l}
1, c_{p, i}\left(t_{i}\right)=j \wedge v_{s, j}\left(t_{i}\right)=i \\
0, c_{p, i}\left(t_{i}\right) \neq j \vee v_{s, j}\left(t_{i}\right) \neq i
\end{array}\right. \\
s t_{v, i}\left(t_{i}\right)=\left\{\begin{array}{c}
1, \exists m_{i, j}\left(t_{i}\right)=1 \wedge m_{i, j}\left(t_{i}\right) \in M \\
0, \nexists m_{i, j}\left(t_{i}\right) \wedge m_{i, j}\left(t_{i}\right) \in M
\end{array}\right. \\
s t_{c, j}\left(t_{i}\right)=\left\{\begin{array}{c}
1, \exists m_{i, j}\left(t_{i}\right)=1 \wedge m_{i, j}\left(t_{i}\right) \in M \\
0, \nexists m_{i, j}\left(t_{i}\right) \wedge m_{i, j}\left(t_{i}\right) \in M
\end{array}\right.
\end{gathered}
$$

The last process is evaluating whether the iteration continues or ends. This evaluation process result is the value of $s t_{m}$. This process is formalized by using Eqs. (35) to (38). Eq. (35) shows that the matching status is stable if the value of all links does not change anymore. Eq. (36) shows that the customer matching status is 1 if all customers receive proposals. Eq. (37) shows that the vehicle matching status is 1 if all vehicles have customers. Eq. (38) is used to determine whether the matching process ends. When the number of customers is less than or equal to the number of vehicles, the matching process ends if all customers have received proposals. Meanwhile, when the number of customers is more than the number of vehicles, the matching process ends if there are not any rejected proposals.

$$
\begin{gathered}
s t_{s}\left(t_{i}\right)=\left\{\begin{array}{l}
1, \forall m_{i, j}\left(t_{i}\right)=m_{i, j}\left(t_{i}-1\right) \\
0, \exists m_{i, j}\left(t_{i}\right) \neq m_{i, j}\left(t_{i}-1\right)
\end{array}\right. \\
s t_{m c}\left(t_{i}\right)=\left\{\begin{array}{l}
1, \nexists s t_{c, j}\left(t_{i}\right)=0 \wedge s t_{s}\left(t_{i}\right)=1 \\
0, \exists s t_{c, j}\left(t_{i}\right)=0 \vee s t_{s}\left(t_{i}\right)=0
\end{array}\right. \\
s t_{m v}\left(t_{i}\right)=\left\{\begin{array}{l}
1, \nexists s t_{v, i}\left(t_{i}\right)=0 \wedge s t_{s}\left(t_{i}\right)=1 \\
0, \exists s t_{v, i}\left(t_{i}\right)=0 \vee s t_{s}\left(t_{i}\right)=0
\end{array}\right. \\
s t_{m}\left(t_{i}\right)=\left\{\begin{array}{l}
s t_{m c}\left(t_{i}\right), n_{c} \leq n_{v} \\
s t_{m v}\left(t_{i}\right), n_{c}>n_{v}
\end{array}\right.
\end{gathered}
$$




\subsection{Simulation}

This dispatching model is then implemented into online taxi dispatch system simulation. In this simulation, the proposed model is compared with the stable marriage [5], limited-movement-grid [9], freemovement-grid [21], circle-shaped-maximumrevenue [17], and circle-shaped-nearest [22] models. These second to fifth models are the agent-based models explained in the related work section.

The environment is a city of Bandung in Indonesia, with the size is approximately $167 \mathrm{~km}^{2}$. This city size is then transformed into $10 \mathrm{~km}$ width and $16.7 \mathrm{~km}$ length. In the beginning, some taxi vehicles and customers are generated. These vehicles and customers are located randomly in this city. This location includes passenger's pickup location, passenger's destination location, and vehicles' current location. The passengers' pickup and vehicles' current locations are in the city central and they are uniformly distributed in the $4 \mathrm{~km}^{2}$ area. The passengers' destination location is uniformly distributed around the city.

Besides these locations, several variables are set randomly. They follow uniform distribution. These processes are formalized by using Eqs. (39) to (42).

$$
\begin{gathered}
s_{v, i}=U\left(s_{v i}, s_{v a}\right) \\
s_{c, j}=U\left(s_{c i}, s_{c a}\right) \\
p_{r, i}=U\left(p_{r i}, p_{m i d}\right) \\
p_{t, i}=U\left(p_{m i d}, p_{t, a}\right)
\end{gathered}
$$

This simulation runs on in a different number of passengers. The number of passengers ranges from 25 persons to 75 persons with the step size is 5 persons. It means that the condition ranges from oversupply to undersupply. There are 30 simulation sessions for every level.

The model performance is evaluated by observing several variables. These variables are average net distance, average pickup distance, average travel cost, average customer satisfaction score, and average vehicle satisfaction score. These variables are evaluated because they are concerned by the driver or the passenger. Meanwhile, the success ratio is used to evaluate the system ability in fulfilling the incoming orders. These observed variables are calculated by using Eqs. (43) to (47). In this simulation, there are several adjusted variables. Default value of these variables are shown in Table 1.
Table 1. Adjusted variables default value

\begin{tabular}{|c|c|}
\hline Variable & Default value \\
\hline$n_{V}$ & 50 unit \\
\hline$W_{n}$ & 1 \\
\hline$W_{p}$ & 1 \\
\hline$W_{b}$ & 1 \\
\hline$W_{c}$ & 100 rupiah \\
\hline$W_{V}$ & 4,500 rupiah \\
\hline$p_{s t e p}$ & 3,000 rupiah \\
\hline$p_{m i d}$ & 6,000 rupiah \\
\hline$p_{r i}$ & 3 \\
\hline$p_{t a}$ & 5 \\
\hline$S_{V m}$ & 1 \\
\hline$S_{V a}$ & 3 \\
\hline$S_{V i}$ & 5 \\
\hline$S_{c m}$ & 1 \\
\hline$S_{c a}$ & \\
\hline$S_{c i}$ & \\
\hline & \\
\hline
\end{tabular}

$$
\begin{gathered}
d_{a v n}=\frac{\sum d_{n, i, j}}{n_{c s}}, \forall(i, j) \in S_{\text {link }, i, j} \\
d_{a v p}=\frac{\sum d_{p, i, j}}{n_{c s}}, \forall(i, j) \in S_{\text {link }, i, j} \\
p_{a t c}=\frac{\sum\left(d_{d, i, j}, p_{c, j}\right)}{n_{c s}} \forall(i, j) \in S_{\text {link }, i, j} \\
s_{a c}=\frac{s_{c, i, j}}{n_{c s}}, \forall(i, j) \in S_{\text {link }, i, j} \\
s_{a v}=\frac{s_{v, i, j}}{n_{c s}}, \forall(i, j) \in S_{\text {link }, i, j}
\end{gathered}
$$

\section{Result and discussion}

Based on the simulation, in this section, we will show and discuss the simulation result. The result is shown in Fig. 2 to Fig. 6. These figures represent the average net distance, average pickup distance, average travel cost, average customer satisfaction score, and average vehicle score consecutively. In every figure, SM represents stable marriage model [5], LMG represents the limited-movement-grid model [9], FMG represents the free-movement-grid model [21], CSMR represents the circle-shapedmaximum-revenue model [17], CSN represents circle-shaped-nearest model [22], and SM-bid represents the proposed stable marriage-first price auction model.

Based on Fig. 2, the average net distance is stagnant in all number of passengers in the CSN [22] and SM [5] models. In the LMG [9] and proposed 


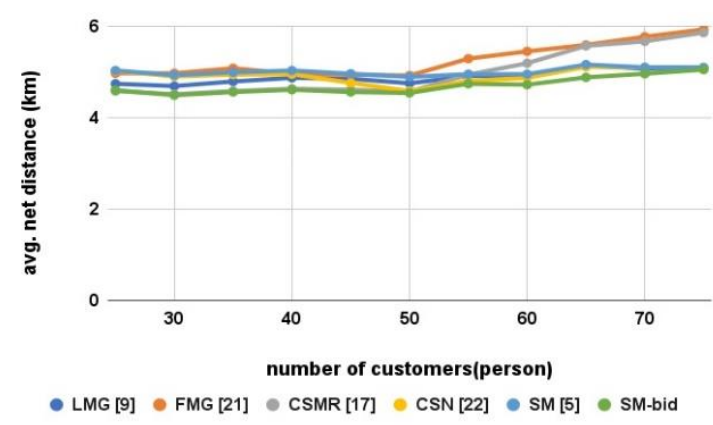

Figure. 2 Average net distance result

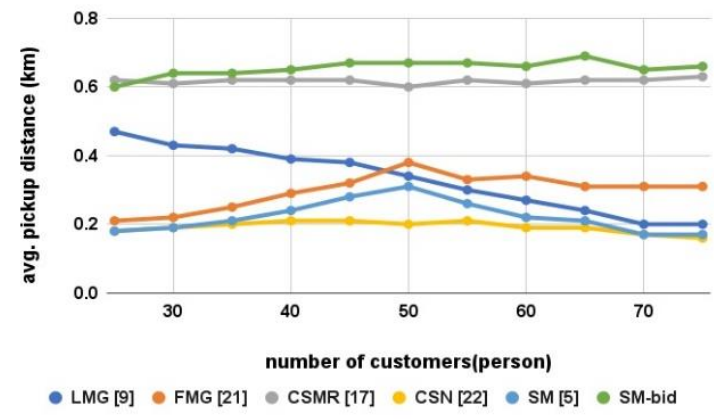

Figure. 3 Average pickup distance result

models, the net distance tends to increase with low inclination due to the increase in demand. In the FMG [21] and CSMR [17] models, the average net distance increases with higher inclination due to the increase in demand. In the overdemand situation, the FMG [21] and CSMR [17] models create the highest average net distance. In the oversupply situation, the CSN [22] and SM [5] models create the highest average net distance. The proposed model tends to create a low average net distance which is 91 percent in the oversupply situation and 85 percent in the overdemand situation compared with the highest average net distance in the same situation.

The explanation of the result in Fig. 3 is as follows. The CSMR [17], CSN [22], the proposed model creates stagnant average pickup distance due to the increase in the demand. In the LMG [9] model, the average pickup distance declines due to the increase in the demand. From the oversupply to equal supplydemand situation, the average pickup distance of the FMG [21] and SM models increases. After that, it tends to stagnant for the FMG [21] model and declines for the SM [5] model. The proposed model creates the highest average pickup distance, which is 3 percent to 4 percent compared with the lowest average pickup distance in the same situation. Fortunately, the average pickup distance of the proposed model is approximately $0.65 \mathrm{~km}$ so that if the vehicle speed is approximately $10 \mathrm{~km}$ per hour, the average pickup time is 3.9 minutes and it still less than 15 minutes.

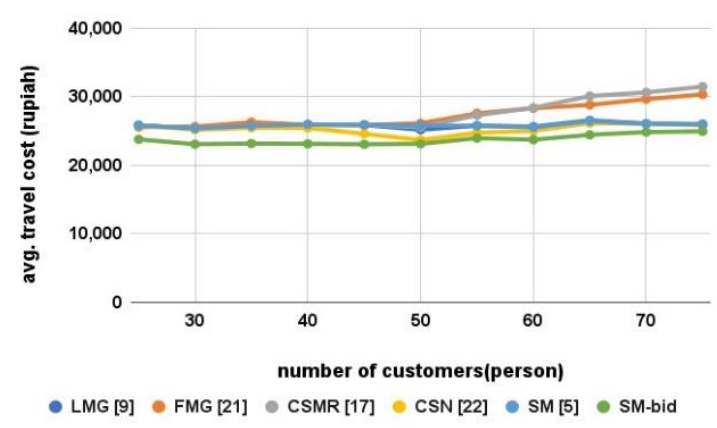

Figure 4. Average travel cost result

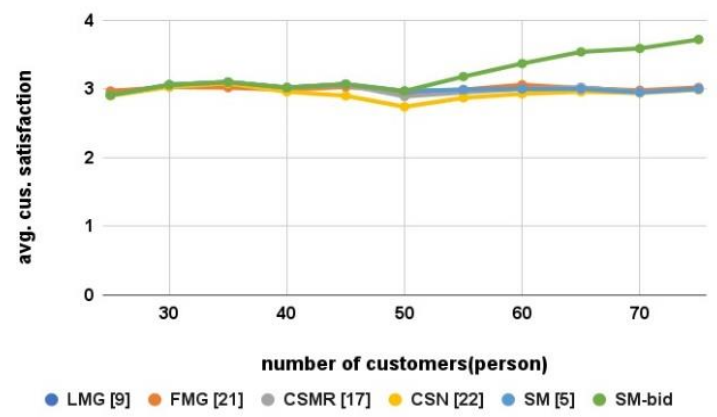

Figure. 5 Average customer satisfaction score result

In Fig. 4, it is shown that the proposed model, due to the auction mechanism, creates lower travel cost rather than all previous models. In the LMG [9], CSN [22], SM [5], and the proposed models, the average travel cost tends to stagnant. Meanwhile, in the FMG [21] and CSMR [17] models, the average travel cost tends to increase due to the increase in the demand. The average travel cost of the proposed model is only 92 percent in the oversupply situation and 79 percent in the overdemand situation compared with the highest average travel cost in the same situation.

In Fig. 5, it is shown that the average customer satisfaction score of the proposed model is the best among the previous models in the undersupply condition. In all previous models, the average customer satisfaction is stagnant. In the proposed model, it is also stagnant in oversupply to equalsupply condition and inclines in the undersupply condition. In the extreme undersupply condition, the average customer satisfaction score of the proposed model is 20 percent better than the previous models. In Fig. 6, it is shown that the average vehicle satisfaction score of the proposed model is the best among all previous models in the oversupply condition. In all previous models, the average vehicle satisfaction is stagnant. In the proposed model, it declines in the oversupply to equal-supply condition and is stagnant in the oversupply condition. In the extreme oversupply condition, the average vehicle satisfaction score of the proposed model is 28 percent higher than the previous models. 


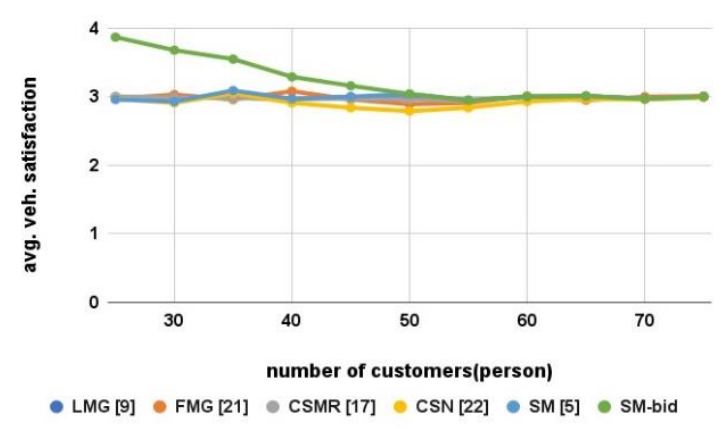

Figure. 6 Average vehicle satisfaction score

Based on this simulation result, there are several findings in the proposed model performance compared with the previous models. By accommodating more parameters, the proposed model sacrifices the pickup distance (waiting time) aspect although it is still in acceptable value. Fortunately, in inequal supply-demand condition, the proposed model can be better in the travel cost, customer satisfaction, and vehicle's satisfaction aspects because these aspects are accommodated in this proposed model. Due to the adoption of the auction-based price mechanism, the passenger is offered lower price rather than the static price. On the other side, the driver whose bidding price is lower has an incentive for a better winning opportunity. On the other side, the driver whose service level is higher also has an incentive in better winning opportunity too. The driver can interpret the service level in many ways, such as driving behaviour, vehicle condition, etc.

Meanwhile, this work has several limitations. In this simulation, there is only one scenario that the passengers and drivers are concentrated in a small area and the passengers' destination is around the wide area so that the net distance tends to big positive. Meanwhile, there are many other scenarios in the real world so that in future work, it will be challenging to evaluate this proposed model in many other scenarios.

Fortunately, this work has demonstrated the dispatching model that accommodates multi variables. It is done by aggregating the weighted score of every variable. As an open model, this model can be modified easily when more variables are included in the model on both passenger's side and/or driver's side.

\section{Conclusion}

To accommodate not the pickup cost and waiting time only, this work has developed a new online taxi dispatching model. Besides pickup distance, these parameters are net distance, driver's bidding price, vehicle's satisfaction score, and customer's satisfaction score. This model is developed by combining the stable marriage model and first-price sealed bid auction to satisfy both the customer and the driver. It also offers competitive travel cost for the customer which is still accepted by the driver. Based on the simulation result, overall, the proposed model performs the best among the previous models, except in the average pickup distance. In the extreme undersupply condition, the proposed model creates 20 percent higher in the average customer's satisfaction score and equal in the average net distance. In the extreme oversupply condition, the proposed model creates 28 percent higher in the vehicle's satisfaction score. The proposed model creates up to 21 percent lower travel cost. Although the proposed model creates the worst in the average pickup distance, the average pickup time is 3.9 minutes with the assumption that the vehicle's speed is $10 \mathrm{~km}$ per hour.

\section{Conflicts of Interest}

The authors declare no conflict of interest.

\section{Author Contributions}

Conceptualization, Purba Daru Kusuma; methodology, Purba Daru Kusuma and Meta Kallista; software, Purba Daru Kusuma; validation, Purba Daru Kusuma and Meta Kallista; formal analysis, Purba Daru Kusuma and Meta Kallista; writingoriginal draft preparation, Purba Daru Kusuma; writing-review and editing, Purba Daru Kusuma and Meta Kallista.

\section{Acknowledgements}

This work was supported by Telkom University, Indonesia.

\section{References}

[1] N. Akbulaev, "The Impact of the Taxi Service Mobile Applications on the Financial Condition of Taxi Companies", International Journal of Scientific \& Technology Research, Vol. 9, No. 2, pp. 2144-2150, 2020.

[2] A. W. Siyal, C. Hongzhuan, and C. Gang, "From Consumer Satisfaction to Recommendation of Mobile App-Based Services: An Overview of Mobile Taxi Booking Apps", SAGE Open, Vol. 11, No. 1, pp. 1-14, 2021.

[3] M. Kummel, F. Busch, and D. Z. W. Wang, "Framework for Automated Taxi Operation: The Family Model", Transportation Research Procedia, Vol. 22, pp. 529-540, 2017. 
[4] P. D. Kusuma, "Nearest Driver-FIFO Combination Model in Online Motorcycle Taxi Dispatch System", Journal of Theoretical and Applied Information Technology, Vol. 95, No. 22, pp. 6236-6247, 2017.

[5] M. Kummel, F. Busch, and D. Z. W. Wang, "Taxi Dispatching and Stable Marriage", Procedia Computer Science, Vol. 83, pp. 163170, 2016.

[6] M. Maciejewski, J. Bischoff, and K. Nagel, "An Assignment-Based Approach to Efficient RealTime City-Scale Taxi Dispatching", IEEE Intelligent Systems, Vol. 31, No. 1, pp. 68-77, 2016.

[7] R. Bai, J. Li, J. A. D. Atkin, and G. Kendall, “A Novel Approach to Independent Taxi Scheduling Problem Based on Stable Matching", Journal of the Operational Research Society, Vol. 65, pp. 1501-1510, 2014.

[8] W. Zhang and Y. Fan, "Spatiotemporal Characteristics and Self-Organization of Urban Taxi Dispatch", Complexity, Vol. 2020, 2020.

[9] Y. Yang, X. Wang, Y. Xu, and Q. Huang, "Multiagent Reinforcement Learning-Based Taxi Predispatching Model to Balance Taxi Supply and Demand", Journal of Advanced Transportation, Vol. 2020, pp. 1-12, 2020.

[10] A. Wicaksono, H. Sulistio, A. W. Wardani, and D. A. Bramiana, "Evaluation of Online Motorcycle Taxi Performance in Surabaya and Sidoarjo Area, Indonesia", Journal of Technology and Social Science, Vol. 1, No. 3, pp. 27-32, 2017.

[11] C. Jing, J. Zhi, S. Yang, and W. Wang, "Impact of Driver Age and Experience in Software Usage on Driving Safety and Usability of CarSharing Software", Journal of Advanced Transportation, Vol. 2021, pp. 1-14, 2021.

[12] J. H. J. Yun, X. Zhao, J. Wu, J. C. Yi, K. B. Park, and W. Y. Jung, "Business Model, Open Innovation, and Sustainability in Car Sharing Industry-Comparing Three Economies", Sustainability, Vol. 12, pp. 1-27, 2020.

[13] M. Liu, E. Brynjoffsson, and J. Dowlatabadi, Technology, Incentives, and Service Quality: the Case of Taxis and Uber, Haas School of Business, University of California, Berkeley, 2018.

[14] X. Qian and S. V. Ukkusuri, "Time-of-Day Pricing in Taxi Markets", IEEE Transaction on Intelligent Transportation Systems, Vol. 18, No. 6, pp. 1610-1622, 2017.

[15] J. V. Hall, J. J. Horton, and D. T. Knoepfle, Pricing in Designed Markets: The Case of RideSharing, 2021.
[16] H. Li and W. Xue, "A Blockchain-Based SealedBid e-Auction Scheme with Smart Contract and Zero-Knowledge Proof", Security and Communication Networks, Vol. 2021, pp. 1-10, 2021.

[17] M. Li, Z. Qin, Y. Jiao, Y. Yang, Z. Gong, J. Wang, C. Wang, G. Wu, and J. Ye, "Efficient Ridesharing Order Dispatching with Mean Field Multi-Agent Reinforcement Learning”, In: Proc. of The Web Conference, 2019.

[18] Y. M. Jin, X. F. Ye, W. L. Liu, T. Wang, and H. Wang, "Dynamic Pricing Model for Cruising Taxicab Based on System Dynamics", Advance in Mechanical Engineering, Vol. 11, No. 2, pp. $1-8,2019$.

[19] A. Glaschenko, A. Ivaschenko, G. Rzevski, and P. Skobelev, "Multi-Agent Real Time Scheduling System for Taxi Companies", In: Proc. of $8^{\text {th }}$ International Conference on Autonomous Agents and Multiagent Systems, 2009.

[20] P. D. Kusuma, "Auction Based Dispatch Model in Online Motorcycle Taxi System", Journal of Theoretical and Applied Information Technology, Vol. 96, No. 18, pp. 6134-6148, 2018.

[21] J. Jin, M. Zhou, W. Zhang, M. Li, Z. Guo, Z. Qin, Y. Jiao, X. Tang, C. Wang, J. Wang, G. Wu, and J. Ye, "CoRide: Joint Order Dispatching and Fleet Management for Multi-Scale Ride-Hailing Platforms", In: Proc. of the $28^{\text {th }} A C M$ International Conference on Information and Knowledge Management (CIKM), 2019.

[22] A. Daoud, F. Balbo, P. Gianessi, and G. Picard, "A Generic Agent Model Towards Comparing Resource Allocation Approaches to On-Demand Transport with Autonomous Vehicles", In: Proc. of the $12^{\text {th }}$ Workshop on Optimization and Learning in Multiagent System, 2021.

[23] D. Gale and L. S. Shapley, "College Admissions and the Stability of Marriage", The American Mathematical Monthly, Vol. 69, No. 1, pp. 9-15, 1962.

[24] Z. Guo, Y. Fu, and C. Cao, "Secure First-Price Sealed-Bid Auction Scheme", EURASIP Journal on Information Security, pp. 1-6, 2017.

[25] F. Deng, S. Liu, and Z. Fang, "First Price Sealed-bid Auction of the Grey Game Improvement Model via Double-Parameter Bayesian Estimation", Grey Systems: Theory and Application, Vol. ahead-of-print, No. aheadof-print, 2021. 